Enferm Bras 2020;19(4);280-9

https://doi.org/10.33233/eb.v19i4.2818

\title{
ARTIGO ORIGINAL \\ Diagnósticos de enfermagem relacionados aos distúrbios do sono em idosos em serviço geriátrico
}

Sanni Moraes de Oliveira*, Wiliana Aparecida Alves de Brito Fernandes, M.Sc. ${ }^{*}$, Maria das Graças Melo Fernandes, D.Sc.*

\author{
*Enfermeira, Universidade Federal da Paraíba, João Pessoa/PB
}

Recebido em 30 de março de 2019; aceito em 7 de agosto de 2020.

Correspondência: Sanni Moraes de Oliveira, Rua Bancário Lourenço Vieira de Souza, 41 Bancários 58051-570 João Pessoa PB

Sanni Moraes de Oliveira: sannidsm@gmail.com

Wiliana Aparecida Alves de Brito Fernandes: wiliana_alves@hotmail.com

Maria das Graças Melo Fernandes: gracaafernandes@hotmail.com

\section{Resumo}

Objetivo: Identificar e caracterizar os Diagnósticos de Enfermagem referentes aos distúrbios do sono em idosos atendidos em um ambulatório geriátrico. Métodos: Trata-se de um estudo quantitativo, do tipo descritivo, transversal, realizado em um hospital universitário, com amostra de 211 idosos, utilizando-se os Diagnósticos de Enfermagem relacionados aos distúrbios do sono da NANDA-I. Resultados: $83,4 \%$ dos idosos investigados apresentaram indicadores clínicos referentes aos Diagnósticos de Enfermagem de distúrbios do sono: Padrão do Sono Prejudicado, Insônia e Privação do Sono. A Insônia foi o Diagnóstico de Enfermagem mais prevalente, sendo as principais características definidoras: a dificuldade para iniciar e manter o sono e o despertar precoce. Conclusão: Os achados demonstram a prevalência significativa dos Diagnósticos de Enfermagem relativos aos distúrbios do sono entre as pessoas idosas. Com vistas à promoção de um sono e repouso adequado a essa população, salienta-se que é imprescindível o enfermeiro proceder com o Processo de Enfermagem buscando identificar os problemas reais e/ou potenciais, promovendo intervenções que garantam a qualidade do sono para esta população.

Palavras-chave: cuidados de enfermagem, idoso; diagnósticos de enfermagem, transtornos do sono-vigília.

\begin{abstract}
Nursing diagnoses related to sleep disorders in elderly people in geriatric service

Objective: To identify and characterize the Nursing Diagnoses related to sleep disorders in the elderly treated at a geriatric outpatient clinic. Methods: This is a quantitative, descriptive, crosssectional study, carried out in a university hospital, with a sample of 211 elderly people, using the Nursing Diagnoses related to NANDA-I sleep disorders. Results: $83.4 \%$ of the elderly investigated had clinical indicators regarding the Nursing Diagnosis of sleep disorders: Impaired Sleep Pattern, Insomnia and Sleep Deprivation. Insomnia was the most prevalent Nursing Diagnosis, with the main defining characteristics: the difficulty to start and maintain sleep and early awakening. Conclusion: The findings demonstrate the significant prevalence of Nursing Diagnoses related to sleep disorders among the elderly. With a view to promoting adequate sleep and rest for this population, it is emphasized that it is essential for nurses to proceed with the Nursing Process, seeking to identify real and / or potential problems, promoting interventions that guarantee the quality of sleep for this population.
\end{abstract}

Keywords: nursing care, aged, nursing diagnosis, sleep wake disorders. 


\section{Resumen \\ Diagnósticos de enfermería relacionados con alteraciones del sueño en personas mayores en servicio geriátrico}

Objetivo: Identificar y caracterizar los Diagnósticos de Enfermería relacionados con trastornos del sueño en ancianos atendidos en un ambulatorio geriátrico. Métodos: Este es un estudio cuantitativo, de tipo descriptivo, transversal, realizado en un hospital universitario, con una muestra de 211 ancianos, utilizando los diagnósticos de enfermería relacionados con los trastornos del sueño de NANDA-I. Resultados: El 83,4\% de los ancianos investigados presentaba indicadores clínicos relacionados con los Diagnósticos de Enfermería de trastornos del sueño: Patrón de sueño deteriorado, Insomnio y Privación del sueño. El insomnio fue el diagnóstico de enfermería más común predominante, siendo las principales características definitorias: la dificultad para iniciar y mantener el sueño y el despertar temprano. Conclusión: Los hallazgos demuestran prevalencia significativa de Diagnósticos de Enfermería relacionados con trastornos del sueño entre los ancianos. Con el fin de promover sueño y descanso adecuados para esta población, se enfatiza que es fundamental que el enfermero proceda con el Proceso de Enfermería, buscando identificar problemas reales y / o potenciales, promoviendo intervenciones que garanticen la calidad del sueño de esta población.

Palabras-clave: atención de enfermería, anciano, diagnósticos de enfermería, trastornos del sueño-vigilia.

Introdução

O envelhecimento se constitui como um processo natural do curso de vida do ser humano, sendo determinado por diversos fatores multidimensionais e complexos que implicam em modificações fisiológicas e causam declínio da homeostasia corporal, aumento da vulnerabilidade às morbidades e comprometimento da saúde do idoso com consequências físicas, mentais ou sociais [1].

Dentre as principais preocupações relacionadas à longevidade estão as ocorrências de condições crônicas, quedas, incapacidade funcional, alterações no sistema nervoso, cardiovascular e musculoesquelético. Além destes, suscita em demandas de cuidados específicos e ressaltam-se as modificações no padrão do sono e suas repercussões negativas para o organismo [2].

O sono por ser uma necessidade fisiológica e apresentar funções restaurativas no organismo e de otimização da energia favorece o equilíbrio das condições físicas e mentais. Com o passar dos anos, as alterações inerentes a senescência trazem repercussões no ciclo do sonovigília tornando-o mais superficial e fragmentado e, portanto, com menor eficiência e pior qualidade. A qualidade do sono é de fundamental importância para prevenção e recuperação de doenças. Uma vez que essa esteja prejudicada poderá desencadear déficits cognitivos, de memória e concentração, desequilíbrio emocional e danos nas funções imunes e inflamatórias com aumento significativo do risco de quedas e culminam para uma diminuição da qualidade de vida, aumento dos índices de morbimortalidade e elevados custos para os serviços de saúde [3].

$\mathrm{Na}$ pessoa idosa, algumas mudanças fisiológicas ocasionam alterações no padrão do sono, geralmente torna-se mais superficial e com despertares frequentes, reduzindo a sensação de descanso, o que acarreta sonolência e cochilos diurnos [2].

Considerando isso, é imprescindível, por parte do enfermeiro, a identificação da qualidade do sono e de possíveis distúrbios relacionados a esse fenômeno, a fim de promover estratégias que visem a preservação e um melhor padrão habitual do sono, por conseguinte, subsidiar a melhora clínica do idoso, refletindo na busca pela integralidade do cuidado a essa parcela da população [3].

Tendo em vista tais necessidades, a North American Nursing Diagnosis Association (NANDA-I, 2015-2017) propõe Diagnósticos de Enfermagem (DE) que podem ser identificados pelo enfermeiro no manejo de pacientes com tais distúrbios, os quais estão locados no Domínio 4, que concerne ao sono/repouso, a partir da coleta de dados e julgamento clínico, os quais são: "Insônia"; "Padrão do sono prejudicado" e; "Privação do sono" [4].

Diante disso, a NANDA-I apresenta o Diagnósticos de Enfermagem "Insônia" definindoo como "distúrbio na qualidade e quantidade do sono que prejudica o desempenho normal das funções da vida diária" [4:384). Quanto ao "Padrão do sono prejudicado" define-o como "Despertares com tempo limitado em razão de fatores externos" [4:387]. E "Privação do Sono" como "Períodos prolongados sem suspensão sustentada natural e periódica do estado de consciência relativa que proporciona o descanso" [4:389]. 
A taxonomia da NANDA-I elenca também as características definidoras dos problemas e os fatores relacionados ou determinantes do evento, o que constitui uma ferramenta importante para a sua identificação e abordagem terapêutica e de cuidado. A despeito disso, há lacunas importantes na produção do conhecimento científico relativo aos problemas de sono nos idosos no campo da Enfermagem.

Considerando essa perspectiva a relevância deste estudo se configura dada a sua potencialidade de gerar informações empíricas importantes relativas aos diferentes aspectos inerentes aos distúrbios do sono em pessoas idosas. Ressalta-se que a identificação dos DE e a implementação de intervenções específicas podem auxiliar os enfermeiros no cuidado à população idosa com má qualidade do sono nos diferentes cenários de prática de atenção à saúde, promovendo, especialmente, melhora em sua qualidade de vida

Ante a problemática em tela, surgiram os seguintes questionamentos: Qual a prevalência dos Diagnósticos de Enfermagem da NANDA-I relativos aos distúrbios do sono entre esses idosos? Quais os indicadores empíricos dos diagnósticos evidenciados pelos idosos atendidos no referido ambulatório? Quais os fatores relacionados ou determinantes dos Diagnósticos de Enfermagem entre esses idosos? Ressalta-se que respostas para essas questões poderão contribuir para o maior conhecimento da Enfermagem acerca desta problemática e para uma melhor assistência diante das necessidades específicas da pessoa idosa com problemas na qualidade de sono.

Para a obtenção de respostas para essas questões, foram delimitados para o estudo os seguintes objetivos: Identificar e caracterizar os DE referentes aos distúrbios do sono em idosos atendidos em um ambulatório geriátrico.

\section{Material e métodos}

Trata-se de estudo com abordagem quantitativa, do tipo descritivo, transversal, desenvolvido em um hospital no município de João Pessoa/PB. A população estudada foi compreendida por pessoas idosas atendidas no ambulatório de Geriatria do referido serviço.

Para a seleção dos idosos investigados, foi levantado junto ao serviço o quantitativo de consultas entre os meses de janeiro de 2017 a junho do mesmo ano, totalizando 664 idosos atendidos. O cálculo do tamanho da amostra se deu a partir da fórmula para populações finitas, levando em consideração o nível de confiança de $95 \%\left(Z_{\infty}=1,96\right)$, o erro amostral de $5 \%$. A determinação da amostra foi do tipo selecionada por conveniência, de forma consecutiva, obtendo-se o quantitativo de 211 entrevistados.

A técnica de amostragem foi não-probabilística, por conveniência. Participaram do estudo pessoas com sessenta anos ou mais, de ambos os sexos. Os critérios de inclusão foram: pessoas idosas vinculadas ao serviço de geriatria; pessoas idosas aguardando atendimento; os critérios de exclusão foram: Idosos que apresentavam limitações graves para compreensão dos questionamentos e aqueles que não apresentavam condições clínicas para responder as perguntas.

A coleta de dados foi realizada no período de novembro de 2017 a maio de 2018, mediante entrevista subsidiada por instrumento estruturado, contemplando questões relativas à caracterização sociodemográfica (sexo, idade, estado civil e renda); e questões dicotômicas abarcando as características definidoras e os fatores relacionados dos DE "Insônia", "Padrão do Sono prejudicado" e "Privação do Sono" da taxonomia NANDA- I, clínico (classificação 20152017), com vistas a facilitar o raciocínio diagnóstico: 1) Análise e síntese dos dados; 2) Estabelecimento dos diagnósticos propriamente ditos [4].

Os dados foram digitados em planilha no Software Microsoft Excel e posteriormente importados ao Statistical Package for the Social Sciences (SPSS), Versão 20.0. A análise dos dados foi efetivada numa abordagem quantitativa por meio da estatística descritiva de natureza univariada para todas as variáveis, incluindo medidas de frequência. E análise bivariada entre as características definidoras dos diagnósticos investigados e as variáveis sociodemográficas, entre as características definidoras/fatores relacionados e sexo, atribuindo um valor de $p<0,05$ para ser considerado como estatisticamente significante (teste bicaudal). O Teste de Qui-Quadrado e - Teste Exato de Fisher foram utilizados para verificar a associação de variáveis categóricas.

Para melhor análise foi aplicado o Índice de Qualidade do Sono de Pittsburg (PSQI). O instrumento contempla sete componentes, os quais somados conferem a pontuação global do PSQI, entre 0 e 21. Pontuações de 0-4 indicam boa qualidade do sono, de 5-10 indicam qualidade ruim e acima de 10 indicam distúrbio do sono. 
Cabe destacar que durante todo o processo da pesquisa, especialmente na fase da coleta de informações empíricas, foram observados os aspectos éticos que normatizam a pesquisa envolvendo seres humanos especialmente o sigilo e a confidencialidade das informações, dispostos na Resolução 466/2012 do CNS/MS/BRASIL, aprovado pelo Comitê de Ética que referenda a instituição, tendo como número de CAE 56053116.8.00005183 e do parecer 508.880 .

\section{Resultados}

Dentre os 211 idosos pesquisados, verificou-se que a maioria pertencia ao sexo feminino, $122(57,8 \%)$, na faixa etária predominante de 60 a 69 anos, $140(66,4 \%)$ e $129(61,1 \%)$ eram casados. Ademais, a maioria, $88(41,7 \%)$ possuía renda familiar de 2 a 3 salários (Tabela I).

Tabela I - Distribuição das variáveis sociodemográficas das pessoas idosas atendidas em serviço de saúde geriátrico, João Pessoa/PB, 2018. $(n=211)$.

\begin{tabular}{llcc}
\hline Variável & Categoria & n & \% \\
\hline Idade & $60-69$ anos & 140 & 66,4 \\
& $70-79$ anos & 55 & 26,1 \\
& $80-89$ & 14 & 6,6 \\
& 90 anos ou mais & 02 & 0,9 \\
\hline \multirow{4}{*}{ Rexo } & Feminino & 122 & 57,8 \\
& Masculino & 89 & 42,2 \\
& Católica & 145 & 68,7 \\
& Evangélica & 55 & 26,1 \\
& Espírita & 07 & 3,3 \\
& Outra & 04 & 1,9 \\
\hline Estado civil & Casado & 129 & 61,1 \\
& Solteiro & 34 & 16,1 \\
& Viúvo & 32 & 15,2 \\
& Divordiado & 16 & 7,6 \\
\hline Renda familiar & Sem renda & 04 & 1,9 \\
& Um salário mínimo & 86 & 40,8 \\
& 2 a 3 salários mínimos & 88 & 41,7 \\
& Mais de 4 salários mínimos & 12 & 5,7 \\
& Não informado & 21 & 10,0 \\
\hline
\end{tabular}

Com relação aos distúrbios do sono, houve a prevalência de 176 idosos $(83,4 \%)$, os quais referiram algum problema, dentre os quais, $142(67,3 \%)$ foram identificados com o DE "Insônia", 97 (46,0\%) apresentaram "Privação de Sono" e 83 (39,3\%) "Distúrbios no padrão do sono" (Tabela II).

Tabela II - Diagnósticos de Enfermagem relativos a distúrbios do sono em idosos atendidos em um ambulatório de geriatria de um hospital universitário em João Pessoa/PB, 2015-2016 ( $n=211)$.

\begin{tabular}{llll}
\hline Variável & & $\mathbf{n}$ & $\%$ \\
\hline Possuem distúrbios do & Sim & 176 & 83,4 \\
sono & Não & 35 & 16,6 \\
& Total & 211 & 100 \\
\hline Diagnósticos de & Insônia & 142 & 67,3 \\
Enfermagem & Privaç̃̃o de Sono & 97 & 46,0 \\
& Padrão do Sono Prejudicado & 83 & 39,3 \\
\hline
\end{tabular}

${ }^{*}$ A soma perfaz um valor superior a amostra em decorrência de um mesmo idoso apresentar mais de um fator relacionado.

Em relação ao DE "Insônia", presente nos idosos investigados, verificou-se que os mesmos evidenciavam um ou mais sintomas ou características definidoras, especialmente: dificuldade para manter o sono $74(20,2 \%)$, acordar cedo demais $69(18,8 \%)$, dificuldade para iniciar o sono $49(13,3 \%)$ e alteração no humor $29(7,9 \%)$. No tocante aos fatores relacionados à Insônia, $88(26,8 \%)$ idosos evidenciaram a ansiedade como principal fator, bem como $73(22,2 \%)$ corresponderam a cochilos frequentes durante o dia, $36(10,9 \%)$ a estressores, $30(9,1 \%)$ a desconforto físico, $25(7,6 \%)$ e $23(7,0 \%)$ devido à depressão, conforme descritos na Tabela III. 
Tabela III - Distribuição das características definidoras e fatores relacionados do Diagnóstico de Enfermagem Insônia em pessoas idosas atendidas em serviço de saúde geriátrico, João Pessoa/PB, 2018. $(n=142)$.

\begin{tabular}{|c|c|c|c|c|c|c|c|}
\hline & \multicolumn{7}{|c|}{0 de Enfermagem: in sônia entre os idosos } \\
\hline Variáveis & $\mathrm{n}$ & $\%$ & $\mathrm{n}$ & $\%$ & $\mathrm{n}$ & $\%$ & \\
\hline Dificuldade para manter o sono & 33 & $(44,6)$ & 41 & $(55,4)$ & 74 & $(20,2)$ & 0,602 \\
\hline Acordar cedo demais & 28 & $(40,6)$ & 41 & $(59,4)$ & 69 & $(18,8)$ & 0,743 \\
\hline Dificuldade para iniciar o sono & 19 & $(38,8)$ & 30 & $(61,2)$ & 49 & $(13,3)$ & 0,582 \\
\hline Alteracão no humor & 10 & $(34,5)$ & 19 & $(65,5)$ & 29 & $(7,9)$ & 0,366 \\
\hline Padrão de sono não restaurador & 6 & $(27,3)$ & 16 & $(72,7)$ & 22 & $(6,0)$ & 0,135 \\
\hline Reducão na qualidade de vida & 6 & $(30,0)$ & 14 & $(70,0)$ & 20 & $(5,4)$ & 0.246 \\
\hline Insatisfação com o sono & 4 & $(21,1)$ & 15 & $(78,9)$ & 19 & $(5,1)$ & 0,051 \\
\hline \multicolumn{8}{|c|}{ Fatores determinantes do Diagnó stico de Enfermagem: insônia entre os idosos } \\
\hline & Fel & & Mas & & Tota & & $p-v a l o r^{*}$ \\
\hline Variáveis & $\mathrm{n}$ & $\%$ & $\mathrm{n}$ & $\%$ & $\mathrm{n}$ & & \\
\hline Ansie: & 32 & $(36,4)$ & 56 & $(63,6)$ & 88 & $(26,8)$ & 0,148 \\
\hline frequentes durante 0 dia & 28 & $(38,4)$ & 45 & $(61,6)$ & 73 & $(22,2)$ & 0,413 \\
\hline Estressores & 14 & $(38,9)$ & 22 & $(61,1)$ & 36 & $(10,9)$ & 0,661 \\
\hline ito físico & 9 & $(30,0)$ & 21 & $(70,0)$ & 30 & $(9,1)$ & 0,145 \\
\hline ambiental & 8 & $(32,0)$ & 17 & $(68,0)$ & 25 & $(7,6)$ & 0.272 \\
\hline Depressão & 9 & & 14 & $\begin{array}{l}(60,9) \\
(00)\end{array}$ & 23 & $(7,0)$ & 0,754 \\
\hline Agente farmacológico & 8 & $(36,4)$ & 14 & $(63,6)$ & 22 & $(6,7)$ & 0,559 \\
\hline
\end{tabular}

*Teste de Qui-quadrado de Pearson p n/s

Verificou-se que o DE "Padrão do sono prejudicado" tem como sua principal característica definidora alteração do padrão de sono com frequência de $88(23,3 \%)$, em seguida a dificuldade para iniciar o sono com 31 (19,0\%), dificuldade de funcionamento diário 29 (17,8\%), despertar não intencional $27(16,6 \%)$. Quanto aos fatores relacionados do diagnóstico supracitado, identificou-se que $41(39,4 \%)$ dos idosos relataram padrão de sono não restaurador, em seguida por barreira ambiental com $33(31,7 \%)$, interrupção causada pelo parceiro de sono $14(13,5 \%)$, privacidade insuficiente $9(8,7 \%)$ e, por último, imobilização $7(6,7 \%)$, expostos na Tabela IV.

Tabela IV - Distribuição das características definidoras e fatores relacionados do Diagnóstico de Enfermagem "Padrão do sono prejudicado" em pessoas idosas atendidas em serviço de saúde geriátrico, João Pessoa/PB, 2018. $(n=83)$.

\begin{tabular}{|c|c|c|c|c|c|c|c|}
\hline \multicolumn{3}{|l|}{ Características definidoras do D } & \multicolumn{5}{|c|}{ Enfermagem: Padrão do sono } \\
\hline & \multicolumn{2}{|c|}{ Fem } & \multicolumn{2}{|c|}{ Masc } & \multicolumn{2}{|c|}{ Total } & $p$-valor \\
\hline Variáveis & $\mathrm{n}$ & $\%$ & $\mathrm{n}$ & $\%$ & $\mathrm{n}$ & $\%$ & \\
\hline Alteração do padrão de sono & 13 & $(34,2)$ & 25 & $(65,8)$ & 38 & $(23,3)$ & $0.272^{*}$ \\
\hline Dificuldade para iniciar o sono & 12 & $(38,7)$ & 19 & $(61,3)$ & 31 & $(19,0)$ & $0,672^{\star}$ \\
\hline Dificuldade de funcionamento diário & & $(36,4)$ & 14 & $(63,6)$ & 22 & $(17,8)$ & $0,559^{\star}$ \\
\hline Despertar não intencional & 8 & $(27,6)$ & 21 & $(72,4)$ & 29 & $(16,6)$ & $0,087^{*}$ \\
\hline Não se sentir descansado & 7 & $(35,0)$ & 13 & $(65,0)$ & 20 & $(12,3)$ & $0.494^{*}$ \\
\hline Insatisfação com o sono & 5 & $(27,8)$ & 13 & $(72,2)$ & 18 & $(11,0)$ & $0,196^{\star}$ \\
\hline \multicolumn{8}{|c|}{$\begin{array}{l}\text { Fato res determin antes do Diagnóstico de Enfermagem: pad rão do sono prejudicado o } \\
\text { entre os idosos }\end{array}$} \\
\hline & \multicolumn{2}{|c|}{ Fem } & \multicolumn{2}{|c|}{ Masc } & \multicolumn{2}{|c|}{ Total } & $p$-valor ${ }^{*}$ \\
\hline Variáveis & $\mathrm{n}$ & $\%$ & $\mathrm{n}$ & & & $\%$ & \\
\hline Padrão de sono não restaurador & 14 & $(34,1)$ & 27 & & 41 & & $0.246^{\star}$ \\
\hline Barreira ambiental & 14 & $(42,4)$ & 19 & $(57,6)$ & 33 & $(31,7)$ & $0,975^{\star}$ \\
\hline Interrupção causada pelo parceiro de & 5 & $(35,7)$ & 9 & $(64,3)$ & 14 & $(13,5)$ & $0,612^{*}$ \\
\hline Privacidade insuficiente & 4 & & 5 & & 09 & & $1,000^{* *}$ \\
\hline Imobilização & 3 & $(42,9)$ & 4 & $(57,1)$ & 07 & $(6,7)$ & $1,000^{\star \star}$ \\
\hline
\end{tabular}

*Teste de Qui-quadrado de Pearson $p$ n/s; **Teste Exato de Fisher $p$ n/s

No tocante ao DE "Privação do Sono", os resultados evidenciaram a ansiedade como característica definidora mais relevante, perfazendo o total de $72(31,6 \%)$ dos entrevistados, seguido por mal-estar, correspondendo a $25(11,0 \%)$ e sonolência com $22(9,6 \%)$. Em relação aos fatores relacionados da "Privação de Sono", encontrou-se o padrão do sono não restaurador 
com $32(22,4 \%)$, pesadelos com $26(18,2 \%)$, desconforto prolongado e mudanças de estágios de sono relacionadas ao envelhecimento ambos com 20 (14,0\%). No Teste de Qui-Quadrado apenas o fator relacionado: Padrão do sono não restaurador mostrou significâncias estatística ( $p$ $=0,012)$, conforme exposto a seguir na Tabela $\mathrm{V}$.

Tabela V - Distribuição das características definidoras e fatores relacionados do Diagnóstico de Enfermagem: Privação do Sono em pessoas idosas atendidas em serviço de saúde geriátrico, João Pessoa/PB, 2018. $(n=97)$.

\begin{tabular}{|c|c|c|c|c|c|c|c|}
\hline 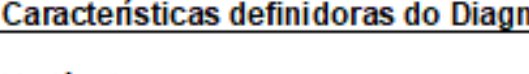 & Fem & & Mas & & $\frac{\text { âo } d}{\text { Tot? }}$ & sono & p-valor ${ }^{*}$ \\
\hline Variáveis & $\mathrm{n}$ & $\%$ & $\mathrm{n}$ & & $\mathrm{n}$ & $\%$ & \\
\hline Ansiedade & 24 & $(33,3)$ & 48 & $(66,7)$ & 72 & $(31,6)$ & 0,061 \\
\hline Mal-estar & 9 & $(36,0)$ & 16 & $(64,0)$ & 25 & $(11,0)$ & 0,505 \\
\hline Sonolência & 10 & $(45,5)$ & 12 & $(54,5)$ & 22 & $(9,6)$ & 0,742 \\
\hline Fadiga & 8 & $(40,0)$ & 12 & $(60,0)$ & 20 & $(8,8)$ & 0,836 \\
\hline Inquietaç̃o & 7 & $(36,8)$ & 12 & $(63,2)$ & 19 & $(8,3)$ & 0,621 \\
\hline Agitação & 6 & $(35,3)$ & 11 & $(64,7)$ & 17 & $(7,5)$ & 0,549 \\
\hline Alteração na concentração & 2 & $(16,7)$ & 10 & $(83,3)$ & 12 & $(5,3)$ & 0,065 \\
\hline \multicolumn{8}{|c|}{ Fatores determinantes do Diagnóstico de Enfermagem: privação do sono } \\
\hline & \multicolumn{2}{|c|}{ Fem } & \multicolumn{2}{|c|}{ Masc } & \multicolumn{2}{|c|}{ Total } & $p$-valor* \\
\hline Variáveis & $\mathrm{n}$ & $\%$ & $\mathrm{n}$ & & $\mathrm{n}$ & $\%$ & \\
\hline Padrão de sono não restaurador & 7 & $(21,9)$ & 25 & $(78,1)$ & 32 & $(22,4)$ & 0,012 \\
\hline Pesadelos & 14 & $(53,8)$ & 12 & $(46,2)$ & 26 & $(18,2)$ & 0,198 \\
\hline Desconforto prolongado & 7 & $(35,0)$ & 13 & $(65,0)$ & 20 & $(14,0)$ & 0,494 \\
\hline Mudanças de estágios de sono & 5 & $(25,0)$ & 15 & $(75,0)$ & 20 & $(14,0)$ & 0,102 \\
\hline $\begin{array}{l}\text { relacionadas ao envelhecimento } \\
\text { Barreira ambiental }\end{array}$ & 5 & $(41,7)$ & 7 & $(58,3)$ & 12 & $(8,4)$ & 0,970 \\
\hline Ambiente excessivamente estimulante & 5 & $(45,5)$ & 6 & $(54,5)$ & 11 & $(7,7)$ & 0,821 \\
\hline
\end{tabular}

Com a aplicação do Teste Qui-quadrado entre as variáveis sociodemográficas e as características definidoras dos diagnósticos investigados, verificou-se que a renda familiar estava associada principalmente a presença de: alterações no padrão do sono $(p<0,001)$; cochilos frequentes durante o dia $(p<0,001)$; alteração na concentração $(p=0,001)$; insatisfação do sono $(p=0,001)$ e privação do sono $(p=0,002)$.

Com vistas a complementar a análise, utilizando-se do Teste de Exato de Fisher, verificou-se uma associação significativa entre renda familiar e qualidade do sono ( $p$-valor $=0,042$ ). As demais características sociodemográficas não apresentaram associação com as variáveis investigadas no presente estudo. Para análise desse resultado, realizou-se a tabulação cruzada da renda familiar com o Índice de Qualidade do Sono de Pittsburg (PSQI), o qual demonstra que a porcentagem total de $59,3 \%$ dos idosos que recebem de 1 a 3 salários mínimos apresentam uma má qualidade do sono, conforme Tabela VI.

Tabela VI - Tabulação cruzada da renda familiar com o Índice de Qualidade do Sono de Pittsburg (PSQI) de pessoas idosas atendidas em serviço de saúde geriátrico, João Pessoa/PB, 2018. (n $=211$.

\begin{tabular}{|c|c|c|c|c|c|c|c|c|}
\hline \multicolumn{3}{|l|}{ Renda familiar } & \multicolumn{6}{|c|}{ PSQI } \\
\hline \multirow{7}{*}{$\begin{array}{l}\text { Variáveis } \\
\text { Sem renda } \\
1 \mathrm{SM} \\
2 \text { a } 3 \mathrm{SM} \\
\text { Mais de } 4 \mathrm{SM} \\
\text { Não informado }\end{array}$} & \multirow{7}{*}{$\begin{array}{l}\mathrm{n} \\
04 \\
86 \\
88 \\
12 \\
21\end{array}$} & \multirow{7}{*}{$\begin{array}{l}\% \\
(1,9) \\
(40,8) \\
(41,7) \\
(5,7) \\
(100)\end{array}$} & \multicolumn{2}{|c|}{$\begin{array}{l}\text { Boa qualidade de } \\
\text { sono }\end{array}$} & \multicolumn{2}{|c|}{$\begin{array}{l}\text { Má qualidade } \\
\text { de sono }\end{array}$} & \multicolumn{2}{|c|}{$\begin{array}{l}\text { Distúrbios do } \\
\text { sono }\end{array}$} \\
\hline & & & $\mathrm{n}$ & $\%$ & $\mathrm{n}$ & $\%$ & $\mathrm{n}$ & $\%$ \\
\hline & & & 0 & $(0,0)$ & 4 & $(1,9)$ & 0 & $(0,0)$ \\
\hline & & & 10 & $(4,7)$ & 66 & $(31,3)$ & 10 & $(4,7)$ \\
\hline & & & 13 & $(6,2)$ & 59 & $(28,0)$ & 16 & $(7,6)$ \\
\hline & & & 5 & $(2,4)$ & 7 & $(3,3)$ & 0 & $(0,0)$ \\
\hline & & & 7 & $(3,3)$ & 10 & $(4,7)$ & 14 & $(1,9)$ \\
\hline
\end{tabular}

*SM = Salário Mínimo $(\mathrm{R} \$ 954,00 / 2018)$

Discussão

No presente estudo, a faixa etária predominante foi entre 60 e 69 anos, havendo uma diminuição gradativa de indivíduos nas faixas subsequentes, especialmente a partir dos 80 anos 
e a maioria dos investigados era casada. Além da expectativa de vida, tal aspecto aponta a maior participação de idosos jovens em atendimento ambulatorial, uma vez que estes geralmente apresentam menores limitações físicas em relação aos mais idosos para frequentarem consultas de rotina [5].

Observou-se que a maioria das pessoas idosas nesta pesquisa foi do sexo feminino, demonstrando não somente a realidade da atual transição demográfica brasileira, denominada de feminização da velhice, como também a maior expectativa de vida entre as mulheres. A participação feminina nas pesquisas com idosos em ambulatório geriátrico pode ser explicada pelo fato de que as mulheres procuram com maior frequência os serviços de saúde ao longo do seu ciclo vital, ante os problemas de saúde que as acometem [6].

A tratar-se dos distúrbios do sono relacionado ao sexo feminino, cabe observar também que as mulheres apresentam maiores alterações neste sentido, pois a tarefa de cuidar é exercida predominante por elas ao longo das suas vidas, dispensando apoio principalmente aos parceiros, bem como outros membros da família [7,8].

O estudo permitiu identificar maior prevalência de DE relacionados aos distúrbios do sono/repouso em pessoas idosas de baixa renda familiar. Esta associação pode despertar uma maior compreensão dos problemas do sono evidenciados neste tipo de comunidade, em situação do nível socioeconômico desfavorável e maior vulnerabilidade social, para que permita ao enfermeiro estabelecer bases para intervenções orientadas para diversos grupos sociais e que sejam adaptadas a realidade do contexto no qual essa pessoa idosa esteja inserida. Neste sentido, deve-se considerar ambiente de dormir inadequado, referente à iluminação, ruídos e temperatura, os quais ocasionam prejuízo em horas de sono por noite, portanto, sono de menor qualidade [9].

Os resultados desta investigação identificaram a prevalência preponderante do $D E$ "Insônia" frente aos demais diagnósticos. As suas características definidoras evidenciadas corroboram os aspectos observados em demais estudos compreendendo principalmente a dificuldade para manter o sono, acordar cedo demais, dificuldade para iniciar o sono, padrão de sono não restaurador e redução na qualidade de vida [10].

Convém destacar que outras características definidoras autorreferidas foram expressas pelos idosos, a exemplo de: sono não restaurador, redução na qualidade de vida, insatisfação com o sono, alteração no afeto, no humor e na concentração, estado de saúde comprometido e energia insuficiente. Tais características corroboram outros estudos e são responsáveis por grande insatisfação, além de prejudicar a saúde física, cognitiva e a qualidade de vida, principalmente, quando esses problemas estão associados às doenças, implicando negativamente na saúde das pessoas, especialmente das idosas [11].

Estudos mostram que a privação do sono em homens saudáveis, durante apenas cinco noites se mostrou suficiente para causar disfunção endotelial venosa e distúrbios do controle autonômico cardiovascular, com aumento significativo na atividade simpática e prejuízo na variabilidade da pressão arterial [3]. Os indicadores clínicos dos DE de distúrbios do sono que produzem consequências no dia seguinte, podem desencadear a privação de sono e prejuízo de sua capacidade de manter a vigília durante o dia. A fragmentação do sono noturno, o aumento da latência para início do sono e a redução de sua duração ocasionada pela dificuldade de se manter dormindo podem ocasionar um aumento da sonolência diurna excessiva [12,13].

Além disso, os cochilos diurnos de longa duração podem ter impacto negativo sobre a qualidade do sono noturno e estar associado a consequências negativas para a saúde, tais como doença cardiovascular, aumento do risco de quedas e de déficits cognitivos [12].

No entanto, torna-se difícil determinar a direção da relação causal entre o cochilo e a perturbação do sono noturno, a qual pode variar entre os indivíduos. Algumas pessoas podem dormir mais durante o dia para compensar uma privação do sono noturno, enquanto outros podem cochilar por outros motivos (por exemplo, inatividade) e, como resultado, desenvolvem dificuldades com o sono noturno [13].

As alterações nos padrões de sono e os sintomas de insônia presentes no envelhecimento podem advir das condições crônicas como de outros distúrbios prevalentes nessa faixa etária, em especial, a depressão, os problemas urinários e neurológicos, a exemplo da doença de Parkinson e o acidente vascular encefálico. Além disso, modificações no cotidiano frequentemente ligadas ao envelhecimento, trazidas pela aposentadoria e a viuvez, podem contribuir para essas alterações também [10].

Salienta-se que o desconforto físico e a má qualidade do sono em idosos muitas vezes podem estar relacionadas à dor, em especial naqueles afetados por condições crônicas de saúde com quadro álgico importante, como artrite e câncer. Porém, a relação entre problemas do sono 
e dor se configura de forma complexa, quando os sintomas podem constituir causa ou consequência um do outro. Sustenta-se, então, a má qualidade do sono do idoso como natural do envelhecimento, e subestima-se a associação entre a dor e o sono prejudicado [10].

A prática de atividade física regular, de acordo com alguns autores, pode ser utilizada como uma intervenção não farmacológica que visa a promoção da qualidade do sono. Atribui-se a melhora observada, ao fato do sono constituir-se numa função biológica voltada para a conservação de energias e a reposição do desgaste tissular cotidiano, as quais aumentam com a prática de exercícios físicos, reduzindo os sintomas de insônia [3]. No entanto, tem-se observado que o tempo destinado às atividades físicas diminui com o passar dos anos, em decorrência das alterações fisiológicas próprias do envelhecimento, da presença de comorbidades e do declínio nas habilidades cognitivas e funcionais. Assim, observa-se que alguns idosos optam por atividades que exigem menor ou nenhum esforço físico, possivelmente por possuírem alguma limitação na sua capacidade funcional [14].

Outro fator relacionado aos DE de distúrbios do sono identificado neste estudo, e que merece destaque, compreende o uso de agentes farmacológicos utilizados pelo idoso. Determinadas classes de medicamentos podem vir a induzir o idoso ao sono, como por exemplo, drogas sedativo-hipnóticas, anti-histamínicos, antidepressivos, e agonistas dopaminérgicos, ocasionando alteração no padrão do sono, contribuindo para a sonolência excessiva diurna. Já as medicações cardiovasculares, neurológicas, psiquiátricas e gastrointestinais também podem estimular excessivamente o idoso, dificultando o início do sono. Destarte, a prescrição de medicamentos a idosos deve ser monitorada, pois alguns medicamentos podem prejudicar a marcha e a cognição desses indivíduos, como também podem causar sonolência e indisposição $[14,15]$.

O profissional de enfermagem possui um importante papel ante as percepções e vivências do paciente, dos seus familiares ou cuidadores. Neste contexto, é fundamental que reconheça a importância da qualidade do sono para as pessoas idosas, para manutenção e recuperação da saúde e qualidade de vida. E durante a assistência, muitas vezes é possível verificar os fatores ambientais e físicos em que estes se inserem, que podem ser intrínsecos do paciente ou extrínsecos, dentre os quais aspectos relacionados a senescência ou senilidade referentes a condição clínica e terapêutica estabelecida, tais como: dor crônica, náuseas, dispneia, apneia do sono, via aérea obstruída, dor/desconforto e frequência urinária, como também fatores psicológicos de ansiedade, angústia, medo ou preocupação. Além disso, fatores extrínsecos relacionados ao ambiente também podem interferir na qualidade do sono de modo fisiológico, os fatores socioeconômicos, espirituais, culturais, recursos disponíveis, condições de higiene, segurança e dinâmica familiar [2,3].

Deste modo, orientações à população acerca da qualidade do sono são relevantes, e intervenções de educação em saúde sobre a temática demandam investimento de esforços dos profissionais envolvidos com a saúde pública. Portanto, a enfermagem, como atividade exercida neste âmbito, deve buscar cada vez mais comprometimento em prestar assistência e programar medidas terapêuticas a fim de melhorar as alterações no sono dos idosos, considerando o processo de perdas próprias do envelhecimento e as possibilidades de manutenção do seu estado de saúde.

Ressalta-se, ainda, a construção e a consolidação do saber próprio da enfermagem para se desenvolver, à medida que utiliza a pesquisa como instrumento do conhecimento teóricoprático. Portanto, a informação recolhida permite intervir de forma mais direcionada e a inferência de DE subsidia sua prática na integralidade no cuidado e no contexto dos transtornos do sono $[4,15]$.

Dado o exposto, as estratégias que podem ser utilizadas para auxiliar ou minimizar as consequências desse problema potencial, devem desconstruir a crença de que a forma mais eficaz de melhorar a qualidade do sono é com intervenção farmacológica. Medidas simples e de baixo custo podem ser oferecidas através de educação em saúde que vise estimular o autocuidado, independência e autonomia por parte dos idosos, conduzindo orientações relativas às medidas de higiene do sono, favorecidas por tecnologias que viabilizem a disseminação de informações e aprendizagem significativas, como hipermídias, jogos, álbuns seriados e cartilhas $[16,17]$.

A higiene do sono se trata da mudança de comportamentos e adequação do ambiente, a fim de favorecer melhoria no sono, sem necessidade de medicamentos. As condutas que integram a higiene do sono perpassam pela alimentação leve antes de deitar, o preparo do corpo e do ambiente para deitar (redução de estímulos sonoros, visuais e luminosos, manutenção de temperatura) e a rotina estabelecida para dormir [18]. 
Outra forma de estimular o sono é incentivar a prática de atividades físicas, a fim de possibilitar o idoso a ser fisicamente ativo e evitar a sonolência diurna. As estratégias de inserção em grupos que desenvolvam atividades visando o restabelecimento de uma rotina na vida do idoso podem contribuir para melhoria do padrão de sono, por meio da regulação do ciclo circadiano. Contudo, a escolha dessas atividades deve ser feita pelos próprios idosos, com o intuito de possibilitar a adesão à sua prática, proporcionando ocupação do tempo livre por uma ação prazerosa e saudável [3].

Outra medida que pode ser considerada é utilização de tampões de ouvidos, máscara de olhos, relaxamento muscular, treinamento de postura e relaxamento, produção sonora. Ainda assim, a música demonstrou-se como um forte aliado para melhoria do padrão de sono, por reduzir ou controlar o estresse e promover o conforto ao atenuar a resposta neuroendócrina do estresse, com consequente diminuição da frequência cardíaca, pressão arterial e frequência respiratória [18-21].

De acordo com a Nursing Intervention Classification, as intervenções de enfermagem podem incluir: orientar o idoso a registrar o padrão do sono e quantidade de horas dormidas; Identificar e reduzir estressores ambientais; Orientar as mudanças no estilo de vida; Conversar com o paciente e a família sobre técnicas que melhorem o sono (medidas de conforto, massagem, posicionamento e toque afetivo); Ensinar ao paciente técnica de relaxamento ou outras formas não farmacológicas de indução do sono; Encorajar uma rotina durante a noite facilitando a transição do estado de alerta ao estado de sono; Reduzir os ruídos evitando luminosidade e sons eletrônicos; Estabelecer rotinas previsíveis para promover os ciclos regulares de sono/vigília; Auxiliar o paciente a limitar o sono durante o dia oferecendo atividades que promovam o estado de vigília, conforme apropriado; Explicar a importância do sono adequado; Determinar os efeitos dos medicamentos do paciente sobre o padrão do sono; Orientar adaptação do ambiente (p. ex., iluminação, ruído, temperatura, colchão e cama) para promover o sono; Monitorar a ingestão de alimentos e as bebidas na hora de dormir em busca de itens que facilitem o sono ou interfiram nele; Iniciar/implementar medidas de conforto, como: Oferecer folhetos com informações sobre técnicas para melhorar o sono [22].

Conclusão

Os achados neste estudo identificaram a alta prevalência dos DE referentes aos distúrbios do sono, principalmente a insônia, comprometendo o início e a manutenção do ciclo circadiano, evidenciando a presença da ansiedade e a sonolência diurna.

Considera-se o papel da enfermagem de suma importância para o levantamento dos principais problemas referente ao sono e repouso entre as pessoas idosas, para que o processo de enfermagem possa ser implementado visando garantir a individualização e a integralidade do cuidado, favorecendo o autocuidado, a independência e autonomia por parte dos idosos.

Sugere-se a realização de estudos científicos que analisem os DE e seus determinantes, bem como analisem a eficácia das intervenções de enfermagem na melhoria da qualidade do sono de pessoas idosas e espera-se que esforços sejam direcionados nos diversos âmbitos de atenção à saúde para o atendimento das reais necessidades das pessoas idosas, com ações que diminuam ou previnam os problemas relacionadas ao sono e repouso, tendo em vista a sua importância para qualidade de vida.

1. Rodrigues RMC, Silva CFR, Loureiro LMJ, Silva SMDT, Crespo SSS, et al. The oldest old: multidimensional functional assessment. Rev Enf Ref 2015;4(5):65-74.

https://doi.org/10.12707/RIV14040

2. Barbosa KTF, Oliveira FMRL, Oliveira SM, Gomes MO, Fernandes MGM. Sleep quality in elderly patients in outpatient care. Rev Enferm UFPE on line 2016;10(2):756-61. https://doi.org/10.5205/reuol.6884-59404-2-SM-1.1002sup201609

3. Andrechuk CRS, Ceolim MF. Sleep quality in patients with acute myocardial infarction. Texto Contexto Enferm 2015;24(4):1104-11. https://doi.org/10.1590/01040707201500002970014

4. Monteiro NT, Ceolim MF. Quality of sleep of the aged at home and in hospital. Texto Contexto Enferm 2014;23(2):356-64. https://doi.org/10.1590/0104-07072014000190013

5. Herdman TH. NANDA International nursing diagnoses: definitions and classification, 2015-2017. Oxford: Wiley-Blackwell; 2015. 
6. Storti LB, Fabrício-Whebe SCC, Kusumota L, Rodrigues RAP, Marques S. Frailty of elderly patients admitted to the medical clinic of an emergency unit at a general tertiary hospital. Texto Contexto Enferm. 2013;22(2):452-9. https://doi.org/10.1590/S010407072013000200022

7. Almeida AV, Mafra SCT, Silva EP, Kanso S. The Feminization of Old Age: a focus on the socioeconomic, personal and family characteristics of the elderly and the social risk. Textos Contextos 2015;14(1):115-31. https://doi.org/10.15448/1677-9509.2015.1.19830

8. Rocha ACO, Mota FRN, Silva MJ, Bonates LAM, Rocha ACO. Quality of life of elderly people who care for the elderly at home Rev Enferm UFPE on line 2015;9(2):548-57. https://doi.org/10.5205/reuol.7028-60723-1-SM.0902201509

9. Tomomitsu MRSV, Perracini MR, Neri AL. Factors associated with satisfaction with life among elderly caregivers and non-caregivers. Ciênc Saúde Coletiva 2014;19(8):342940. https://doi.org/10.1590/1413-81232014198.13952013

10. Lallukkaa T, Ferrie JE, Kivimäki M, Shipley MJ, Rahkonen O, Lahelmaa E. Economic difficulties and subsequent sleep problems: Evidence from British and Finnish occupational cohorts. Sleep Medicine 2012;13(6):680-5. https://doi.org/10.1016/i.sleep.2011.10.036

11. Santos AA, Neri AL, Ceolim MF. Association between insomnia and rheumatoid arthritis in elderly. Rev Esc Enferm USP 2013;47(4):869-75. https://doi.org/10.1590/S0080623420130000400014

12. Kubota AMA, Silva MNRMO, Masioli AB. Aspectos da insônia no adulto e a relação com o desempenho ocupacional. Rev Ter Ocup Univ São Paulo 2014;25(2):119-25. https://doi.org/10.11606/issn.2238-6149.v25i2p119-25

13. Santos AA, Mansano-Schlosser TCS, Ceolim MF, Pavarini SCI. Sleep, fragility and cognition: a multicenter study with Brazilian elderly. Rev Bras Enferm 2013;66(3):351-7. https://doi.org/10.1590/S0034-71672013000300008

14. Mansano-Schlosser TC, Santos AA, Camargo SOR, Freitas DCCV, Lorenz VR, Ceolim MF. Institutionalized elderly: chronological organization of daily routines and sleep quality. Rev Bras Enferm 2014;67(4):610-6. https://doi.org/10.1590/00347167.2014670417

15. Santos AA, Ceolim MF, Pavarini SCI, Neri AL, Rampazo MC Association between sleep disorders and frailty status among elderly. Acta Paul Enferm 2014;27(2):120-5. https://doi.org/10.1590/1982- 0194201400022

16. Valcarenghi RV, Santos SSC, Hammerschmidt KSA, Barlem ELD, Gomes GC, Silva BT. Ações institucionais alicerçadas em diagnósticos de enfermagem para prevenção de quedas em idosos. Rev Rene 2014;15(2):224-32. https://doi.org/10.15253/21756783.2014000200006

17. Sousa CA, Cesar CLG, Barros MBA, Carandina L, Goldbaum M, Marchioni DML, et al. Prevalence of leisure-time physical activity and associated factors: a population-based study in São Paulo, Brazil, 2008-2009. Cad Saúde Pública 2013;29(2):270-82. https://doi.org/10.1590/S0102-311X2013000200014

18. Mesquita CK, Fortes FML, Miguel GNN, Moura SGG. Construção e validação de cartilha para idoso acerca da higiene do sono. Rev Bras Enferm 2019;72(Suppl 2): 214220. https://doi.org/10.1590/0034-7167-2018-0603

19. Correa LP, Braga TR, Malaquias LC, Bessa MEP, Marques MB. Intervenções de enfermagem nos cuidados aos pacientes idosos com Alzheimer: revisão integrativa. Rev Enferm UFPI 2016;5(1):84-8. https://doi.org/10.26694/reufpi.v5i1.3000

20. Nóbrega RV, Nóbrega MML, Silva KL. Nursing diagnoses, outcomes and interventions for children in a Pediatric Clinic of a school hospital. Rev Bras Enferm Brasília 2011;64(3):501-10. https://doi.org/10.1590/S0034-71672011000300014

21. Souza MF, Silva SRC, Brito PV, Siqueira CAL. Intervenções não farmacológicas para o sono de pacientes submetidos à cirurgia cardíaca: revisão sistemática. Rev Latino-Am Enferm 2017;25:e2926. https://doi.org/10.1590/1518-8345.1917.2926

22. Bulechek GM, Butcher HK, Dochterman JM. Classificação das intervenções de enfermagem - NIC. $5^{\text {a }}$ ed. Rio de Janeiro: Elsevier; 2010. 944p. 